\title{
On Cartesian Products of Orthogonal Double Covers
}

\author{
R. El Shanawany, M. Higazy, and A. El Mesady \\ Department of Physics and Engineering Mathematics, Faculty of Electronic Engineering, Menoufiya University, \\ Menouf 32952, Egypt \\ Correspondence should be addressed to A. El Mesady; ahmed_mesady88@yahoo.com
}

Received 9 December 2012; Revised 11 February 2013; Accepted 18 March 2013

Academic Editor: Ilya M. Spitkovsky

Copyright (C) 2013 R. El Shanawany et al. This is an open access article distributed under the Creative Commons Attribution License, which permits unrestricted use, distribution, and reproduction in any medium, provided the original work is properly cited.

Let $H$ be a graph on $n$ vertices and $\mathscr{G}$ a collection of $n$ subgraphs of $H$, one for each vertex, where $\mathscr{G}$ is an orthogonal double cover (ODC) of $H$ if every edge of $H$ occurs in exactly two members of $\mathscr{G}$ and any two members share an edge whenever the corresponding vertices are adjacent in $H$ and share no edges whenever the corresponding vertices are nonadjacent in $H$. In this paper, we are concerned with the Cartesian product of symmetric starter vectors of orthogonal double covers of the complete bipartite graphs and using this method to construct ODCs by new disjoint unions of complete bipartite graphs.

\section{Introduction}

For the definition of an orthogonal double cover (ODC) of the complete graph $K_{n}$ by a graph $G$ and for a survey on this topic, see [1]. In [2], this concept has been generalized to ODCs of any graph $H$ by a graph $G$.

While in principle any regular graph is worth considering (e.g., the remarkable case of hypercubes has been investigated in [2]), the choice of $H=K_{n, n}$ is quite natural, and also in view of a technical motivation, ODCs of such graphs are a helpful tool for constructing ODCs of $K_{n}$ (see [3, page 48]).

In this paper, we assume $H=K_{n, n}$, the complete bipartite graph with partition sets of size $n$ each.

An ODC of $K_{n, n}$ is a collection $\mathscr{G}=\left\{G_{0}, G_{1}, \ldots, G_{n-1}\right.$, $\left.F_{0}, F_{1}, \ldots, F_{n-1}\right\}$ of $2 n$ subgraphs (called pages) of $K_{n, n}$ such that

(i) every edge of $K_{n, n}$ is in exactly one page of $\left\{G_{0}, G_{1}\right.$, $\left.\ldots, G_{n-1}\right\}$ and in exactly one page of $\left\{F_{0}, F_{1}, \ldots, F_{n-1}\right\}$;

(ii) for $i, j \in\{0,1,2, \ldots, n-1\}$ and $i \neq j, E\left(G_{i}\right) \cap E\left(G_{j}\right)=$ $E\left(F_{i}\right) \cap E\left(F_{j}\right)=\emptyset$; and $\left|E\left(G_{i}\right) \cap E\left(F_{j}\right)\right|=1$ for all $i, j \in\{0,1,2, \ldots, n-1\}$.

If all the pages are isomorphic to a given graph $G$, then $\mathscr{G}$ is said to be an ODC of $K_{n, n}$ by $G$.

Denote the vertices of the partition sets of $K_{n, n}$ by $\left\{0_{0}, 1_{0}, \ldots,(n-1)_{0}\right\}$ and $\left\{0_{1}, 1_{1}, \ldots,(n-1)_{1}\right\}$. The length of an edge $x_{0} y_{1}$ of $K_{n, n}$ is defined to be the difference $y-x$, where $x, y \in \mathbb{Z}_{n}=\{0,1,2, \ldots, n-1\}$. Note that sums and differences are calculated in $\mathbb{Z}_{n}$ (i.e., sums and differences are calculated modulo $n$ ).

Throughout the paper we make use of the usual notation: $K_{m, n}$ for the complete bipartite graph with partition sets of sizes $m$ and $n, P_{n}$ for the path on $n$ vertices, $C_{n}$ for the cycle on $n$ vertices, $K_{n}$ for the complete graph on $n$ vertices, $K_{1}$ for an isolated vertex, $G \cup H$ for the disjoint union of $G$ and $H$, and $m G$ for $m$ disjoint copies of $G$.

An algebraic construction of ODCs via "symmetric starters" (see Section 2) has been exploited to get a complete classification of ODCs of $K_{n, n}$ by $G$ for $n \leq 9$, a few exceptions apart, all graphs $G$ are found this way (see [3, Table 1]). This method has been applied in $[3,4]$ to detect some infinite classes of graphs $G$ for which there are ODCs of $K_{n, n}$ by $G$.

In [5], Scapellato et al. studied the ODCs of Cayley graphs and they proved the following. (i) All 3-regular Cayley graphs, except $K_{4}$, have ODCs by $P_{4}$. (ii) All 3-regular Cayley graphs on Abelian groups, except $K_{4}$, have ODCs by $P_{3} \cup K_{2}$. (iii) All 3-regular Cayley graphs on Abelian groups, except $K_{4}$ and the 3-prism (Cartesian product of $C_{3}$ and $K_{2}$ ), have ODCs by $3 K_{2}$.

Much research on this subject focused on the detection of ODCs with pages isomorphic to a given graph $G$. For a summary of results on ODCs, see $[1,4]$. The other terminologies not defined here can be found in [6]. 


\section{Symmetric Starters}

All graphs here are finite, simple, and undirected. Let $\Gamma=$ $\left\{\gamma_{0}, \ldots, \gamma_{n-1}\right\}$ be an (additive) abelian group of order $n$. The vertices of $K_{n, n}$ will be labeled by the elements of $\Gamma \times \mathbb{Z}_{2}$. Namely, for $(v, i) \in \Gamma \times \mathbb{Z}_{2}$ we will write $v_{i}$ for the corresponding vertex and define $\left\{w_{i}, u_{j}\right\} \in E\left(K_{n, n}\right)$ if and only if $i \neq j$, for all $w, u \in \Gamma$ and $i, j \in \mathbb{Z}_{2}$. If there is no chance of confusion, $(w, u)$ will be written instead of $\left\{w_{0}, u_{1}\right\}$ for the edge between the vertices $w_{0}, u_{1}$.

Let $G$ be a spanning subgraph of $K_{n, n}$ and let $a \in \Gamma$. Then the graph $G+a$ with $E(G+a)=\{(u+a, v+a):(u, v) \in$ $E(G)\}$ is called the a-translate of $G$. The length of an edge $e=$ $(u, v) \in E(G)$ is defined by $d(e)=v-u$.

$G$ is called a half starter with respect to $\Gamma$ if $|E(G)|=n$ and the lengths of all edges in $G$ are mutually distinct; that is, $\{d(e): e \in E(G)\}=\Gamma$. The following three results were established in [3].

Theorem 1. If $G$ is a half starter, then the union of all translates of $G$ forms an edge decomposition of $K_{n, n}$; that is, $\bigcup_{a \in \Gamma} E(G+$ a) $=E\left(K_{n, n}\right)$.

Hereafter, a half starter $G$ will be represented by the vector $v(G)=\left(v_{\gamma_{0}}, \ldots, v_{\gamma_{n-1}}\right)$, where $v_{\gamma_{i}} \in \Gamma$ and $\left(v_{\gamma_{i}}\right)_{0}$ is the unique vertex $\left(\left(v_{\gamma_{i}}, 0\right) \in \Gamma \times\{0\}\right)$ that belongs to the unique edge of length $\gamma_{i}$ in $G$.

Two half starter vectors $v\left(G_{0}\right)$ and $v\left(G_{1}\right)$ are said to be orthogonal if $\left\{v_{\gamma}\left(G_{0}\right)-v_{\gamma}\left(G_{1}\right): \gamma \in \Gamma\right\}=\Gamma$.

Theorem 2. If two half starter vectors $v\left(G_{0}\right)$ and $v\left(G_{1}\right)$ are orthogonal, then $G=\left\{G_{a, i}:(a, i) \in \Gamma \times \mathbb{Z}_{2}\right\}$ with $G_{a, i}=G_{i}+a$ is an ODC of $K_{n, n}$.

The subgraph $G_{s}$ of $K_{n, n}$ with $E\left(G_{s}\right)=\left\{\left(u_{0}, v_{1}\right):\left(v_{0}, u_{1}\right) \epsilon\right.$ $E(G)\}$ is called the symmetric graph of $G$. Note that if $G$ is a half starter, then $G_{s}$ is also a half starter.

A half starter $G$ is called a symmetric starter with respect to $\Gamma$ if $v(G)$ and $v\left(G_{s}\right)$ are orthogonal.

Theorem 3. Let $n$ be a positive integer and let $G$ be a half starter represented by the vector $v(G)=\left(v_{\gamma_{0}}, \ldots, v_{\gamma_{n-1}}\right)$. Then $G$ is symmetric starter if and only if $\left\{v_{\gamma}-v_{-\gamma}+\gamma: \gamma \in \Gamma\right\}=\Gamma$.

The above results on ODCs of graphs motivated us to consider ODCs of $K_{m n, m n}$ if we have the ODCs of $K_{n, n}$ by $G$ and ODCs of $K_{m, m}$ by $H$ where $G, H$ are symmetric starters. In this paper, we have settled the existence problem of ODCs of $K_{m n, m n}$ by few infinite families of graphs presented in the next section.

\section{The Main Results}

In the following, if there is no danger of ambiguity, if $(i, j) \in$ $\mathbb{Z}_{n} \times \mathbb{Z}_{m}$ we can write $(i, j)$ as $i j$.

Theorem 4. The Cartesian product of any two symmetric starter vectors is a symmetric starter vector with respect to the Cartesian product of the corresponding groups.
Proof. Let $v(G)=\left(v_{0}, v_{1}, \ldots, v_{n-1}\right) \in \mathbb{Z}_{n}^{n}$ be a symmetric starter vector of an ODC of $K_{n, n}$ by $G$ with respect to $\mathbb{Z}_{n}$, then

$$
\left\{v_{i}-v_{-i}+i: i \in \mathbb{Z}_{n}\right\}=\mathbb{Z}_{n}
$$

Let $u(H)=\left(u_{0}, u_{1}, \ldots, u_{m-1}\right) \in \mathbb{Z}_{m}^{m}$ be a symmetric starter vector of an ODC of $K_{m, m}$ by $H$ with respect to $\mathbb{Z}_{m}$, then

$$
\left\{u_{j}-u_{-j}+j: j \in \mathbb{Z}_{m}\right\}=\mathbb{Z}_{m}
$$

Then $\left.v(G) \times u(H)=\left(v_{0} u_{0}, v_{0} u_{1}, \ldots, v_{i} u_{j}, \ldots, v_{n-1} u_{m-1}\right)\right)$ where $i \in \mathbb{Z}_{n}$ and $j \in \mathbb{Z}_{m}$.

From (1) and (2), we conclude

$$
\begin{aligned}
& \left\{v_{i} u_{j}-v_{-i} u_{-j}+i j: i j \in \mathbb{Z}_{n} \times \mathbb{Z}_{m}\right\} \\
& =\left\{\left(v_{i}-v_{-i}+i\right)\left(u_{j}-u_{-j}+j\right): i \in \mathbb{Z}_{n},\right. \\
& \left.\quad j \in \mathbb{Z}_{m}, i j \in \mathbb{Z}_{n} \times \mathbb{Z}_{m}\right\} \\
& =\mathbb{Z}_{n} \times \mathbb{Z}_{m} .
\end{aligned}
$$

Then $v(G) \times u(H)$ is a symmetric starter vector of an ODC of $K_{m n, m n}$, with respect to $\mathbb{Z}_{n} \times \mathbb{Z}_{m}$, by a new graph $G \times H$ which can be described as follows.

Since $E(G)=\left\{\left(v_{i}, v_{i}+i\right): i \in \mathbb{Z}_{n}\right\}$ and $E(H)=\left\{\left(u_{j}, u_{j}+j\right)\right.$ : $\left.j \in \mathbb{Z}_{m}\right\}$, then $E(G \times H)=\left\{\left(v_{i} u_{j}, v_{i} u_{j}+i j\right): i j \in \mathbb{Z}_{n} \times \mathbb{Z}_{m}\right\}$. It should be noted that $G \times H$ is not the usual Cartesian product of the graphs $G$ and $H$ that has been studied widely in the literature.

All our results based on the following two major points:

(1) the cartesian product construction in Theorem 4

(2) The existence of symmetric starters for a few classes of graphs that can be used as ingredients for cartesian product construction to obtain new symmetric starters. These are as follows.

(1) $K_{1, n}$ which is a symmetric starter of an ODC of $K_{n, n}$ whose vector is $v\left(K_{1, n}\right)=(\overbrace{0,0,0, \ldots, 0}^{n \text { times }} \epsilon$ $\mathbb{Z}_{n}^{n}$, see Corollary 2.2.7 in [7].

(2) $m K_{2,2}$ which is a symmetric starter of an ODC of $K_{4 m, 4 m}$ whose vector is $v\left(m K_{2,2}\right)=$ $(0,1,2, \ldots, 2 m-1,0,1,2, \ldots, 2 m-1) \in \mathbb{Z}_{4 m}^{4 m}$, see [7, Lemma 2.2.13].

(3) $K_{1,2} \cup K_{1,2(n-1)}$ which is a symmetric starter of an ODC of $K_{2 n, 2 n}$ whose vector is $v\left(K_{1,2} \mathrm{U}\right.$ $\left.K_{1,2(n-1)}\right)=(0, \overbrace{n, n, \ldots, n, n}^{(n-1) \text { times }}, 0, \overbrace{n, n, \ldots, n, n)}^{(n-1) \text { times }} \in$ $\mathbb{Z}_{2 n}^{2 n}, n \geq 2$, and it is easily checked that $v_{i}\left(K_{1,2} \cup\right.$ $\left.K_{1,2(n-1)}\right)=v_{-i}\left(K_{1,2} \cup K_{1,2(n-1)}\right)$, and hence $\left\{v_{i}-\right.$ $\left.v_{-i}+i: i \in \mathbb{Z}_{2 n}\right\}=\mathbb{Z}_{2 n}$. 
(4) $K_{1,2} \cup K_{2, n-1}$ which is a symmetric starter of an ODC of $K_{2 n, 2 n}$ whose vector is $v\left(K_{1,2} \cup K_{2, n-1}\right)=$ $(0, \overbrace{1,1,1, \ldots, 1,1}^{(n-1) \text { times }}, n \overbrace{n+1, n+1, \ldots, n+1}^{(n-1) \text { times }}) \in$ $\mathbb{Z}_{2 n}^{2 n}, n \geq 2$, for this vector, and it is easily checked that

$v_{i}-v_{-i}+i= \begin{cases}i & \text { if } i=0, n, \text { or } \\ n+i & \text { otherwise }\end{cases}$

and hence $\left\{v_{i}-v_{-i}+i: i \in \mathbb{Z}_{2 n}\right\}=\mathbb{Z}_{2 n}$.

(5) $K_{2,3}$ which is a symmetric starter of an ODC of $K_{6,6}$ whose vector is $v\left(K_{2,3}\right)=(0,0,3,3,3,0) \epsilon$ $\mathbb{Z}_{6}^{6}$, see [4, Theorem 2.2.7].

These known symmetric starters will be used as ingredients for the cartesian product construction to obtain new symmetric starters.

Theorem 5. For all positive integers $m, n$ with $\operatorname{gcd}(m, 3)=1$, there exists an $O D C$ of $K_{4 m n, 4 m n}$ by $m K_{2,2 n} \cup 2 m(3 n-1) K_{1}$.

Proof. Since $v\left(m K_{2,2}\right)$ and $v\left(K_{1, n}\right)$ are symmetric starter vectors, then $v\left(m K_{2,2}\right) \times v\left(K_{1, n}\right)$ is a symmetric starter vector with respect to $\mathbb{Z}_{4 m} \times \mathbb{Z}_{n}$ (Theorem 4 ). The resulting symmetric starter graph has the following edges set:

$$
\begin{aligned}
E( & \left(m K_{2,2 n}\right) \\
& =\bigcup_{i=0}^{m-1}\left\{\begin{array}{c}
(0 i, \beta(2 i)),(0 i, 2 \beta(m+i)),(0(m+i), \beta(2 i)), \\
(0(m+i), 2 \beta(m+i): 0 \leq \beta \leq n-1
\end{array}\right\} .
\end{aligned}
$$

Lemma 6. For any positive integer $m$ with $\operatorname{gcd}(m, 3)=1$, there exists an $O D C$ of $K_{16 m, 16 m}$ by $m K_{4,4} \cup 24 m K_{1}$.

Proof. Since $v\left(K_{2,2}\right)$ and $v\left(m K_{2,2}\right)$ are symmetric starter vectors, then $v\left(K_{2,2}\right) \times v\left(m K_{2,2}\right)$ is a symmetric starter vector with respect to $\mathbb{Z}_{4} \times \mathbb{Z}_{4 m}$ (Theorem 4 ), and the resulting symmetric starter graph has the following edges set:

$$
\begin{aligned}
& E\left(m K_{4,4}\right) \\
& \left.=\bigcup_{l=0}^{m-1}\left\{\begin{array}{c}
\left\{\begin{array}{c}
\{(0 l, 0 l+i j): i j \in\{0 l, 0(l+2 m), 2 l, 2(l+2 m)\}\} \cup \\
(0(l+m), 0(l+m)+i j): \\
i j \in\{0(l+m), 0(l+3 m), 2(l+m), 2(l+3 m)\}
\end{array}\right\} \\
\left\{\begin{array}{c}
\{(1 l, 1 l+i j): i j \in\{1 l, 1(l+2 m), 3 l, 3(l+2 m)\}\} \\
(1(l+m), 1(l+m)+i j):
\end{array}\right. \\
\{i j \in\{1(l+m), 1(l+3 m), 3(l+m), 3(l+3 m)\}
\end{array}\right\}\right\} .
\end{aligned}
$$

Lemma 7. For any positive integer $m$ with $\operatorname{gcd}(m, 3)=1$, there exists an $O D C$ of $K_{32 m, 32 m}$ by $2 m K_{4,4} \cup 48 m K_{1}$.

Proof. Since $v\left(2 K_{2,2}\right)$ and $v\left(m K_{2,2}\right)$ are symmetric starter vectors, then $v\left(2 K_{2,2}\right) \times v\left(m K_{2,2}\right)$ is a symmetric starter vector with respect to $\mathbb{Z}_{8} \times \mathbb{Z}_{4 m}$ (Theorem 4 ), and the resulting symmetric starter graph has the following edges set:

$$
\begin{aligned}
& E\left(2 m K_{4,4}\right)
\end{aligned}
$$

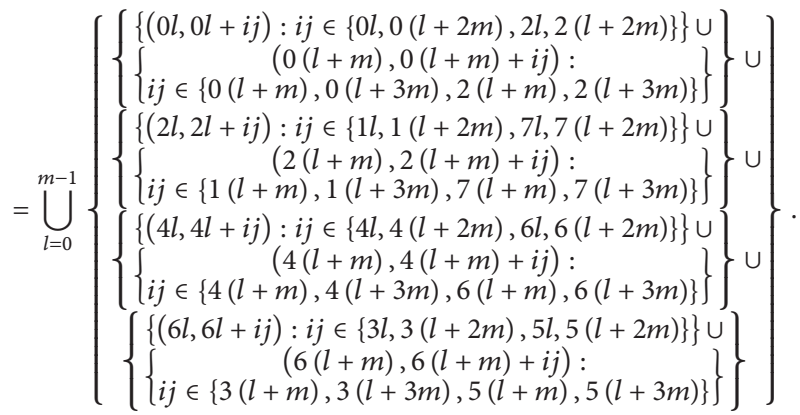

The following conjecture generalizes Lemmas 6 and 7.

Conjecture 8. For all positive integers $m, n$ with $\operatorname{gcd}(m, 3)=$ 1 and $\operatorname{gcd}(n, 3)=1$, there exists an ODC of $K_{16 m n, 16 m n}$ by $n m K_{4,4} \cup 24 m n K_{1}$.

Theorem 9. For all positive integers $m, n \geq 2$, there exists an $O D C$ of $K_{2 m n, 2 m n}$ by $K_{1,2 m} \cup K_{1,2 m(n-1)} \cup 2(m n-1) K_{1}$.

Proof. Since $v\left(K_{1, m}\right)$ and $v\left(K_{1,2} \cup K_{1,2(n-1)}\right)$ are symmetric starter vectors, then $v\left(K_{1, m}\right) \times v\left(K_{1,2} \cup K_{1,2(n-1)}\right)$ is a symmetric starter vector with respect to $\mathbb{Z}_{m} \times \mathbb{Z}_{2 n}$ (Theorem 4 ), and the resulting symmetric starter graph has the following edges set:

$$
\begin{aligned}
E & \left(K_{1,2 m} \cup K_{1,2 m(n-1)}\right) \\
& =\bigcup_{\alpha=0}^{m-1}\left\{(00, \alpha \beta),(0 n, \alpha \gamma): \beta \in\{0, n\}, \gamma \in \mathbb{Z}_{2 n} \backslash\{n\}\right\} .
\end{aligned}
$$

Theorem 10. For all positive integers $m, n \geq 2$, there exists an ODC of $K_{4 m n, 4 m n}$ by $K_{1,4} \cup K_{1,4(n-1)} \cup K_{1,4(m-1)} \cup$ $K_{1,4(m-1)(n-1)} \cup 4(m n-1) K_{1}$.

Proof. Since $v\left(K_{1,2} \cup K_{1,2(m-1)}\right)$ and $v\left(K_{1,2} \cup K_{1,2(n-1)}\right)$ are symmetric starter vectors, then $v\left(K_{1,2} \cup K_{1,2(m-1)}\right) \times v\left(K_{1,2} \cup\right.$ $\left.K_{1,2(n-1)}\right)$ is a symmetric starter vector with respect to $\mathbb{Z}_{2 m} \times$ $\mathbb{Z}_{2 n}$ (Theorem 4 ), and the resulting symmetric starter graph has the following edges set:

$$
\begin{aligned}
E & \left(K_{1,4} \cup K_{1,4(n-1)} \cup K_{1,4(m-1)} \cup K_{1,4(m-1)(n-1)}\right) \\
& =\bigcup_{k=1, k \neq m}^{2 m-1}\left\{\begin{array}{c}
(00, \alpha \beta),(0 n, \alpha \gamma),(m 0, k \beta),(m n, k \gamma): \\
\alpha \in\{0, m\}, \beta \in\{0, n\}, \gamma \in \mathbb{Z}_{2 n} \backslash\{0, n\}
\end{array}\right\} .
\end{aligned}
$$

Theorem 11. For all positive integers $m, n \geq 2$ with $\operatorname{gcd}(m, 3)=1$, there exists an ODC of $K_{8 m n, 8 m n}$ by $m K_{2,4} \cup$ $m K_{2,4(n-1)} \cup 4 m(3 n-1) K_{1}$. 
Proof. Since $v\left(K_{1,2} \cup K_{1,2(n-1)}\right)$ and $v\left(m K_{2,2}\right)$ are symmetric starter vectors, then $v\left(K_{1,2} \cup K_{1,2(n-1)}\right) \times v\left(m K_{2,2}\right)$ is a symmetric starter vector with respect to $\mathbb{Z}_{2 n} \times \mathbb{Z}_{4 m}$ (Theorem 4 ), and the resulting symmetric starter graph has the following edges set:

$$
\begin{aligned}
& E\left(m K_{2,4} \cup m K_{2,4(n-1)}\right) \\
& =\bigcup_{l=0}^{m-1}\left\{\begin{array}{c}
\left\{\begin{array}{c}
\{(0 l, 0 l+i j): i j \in\{0 l, 0(l+2 m), 2 l, 2(l+2 m)\}\} \cup \\
\left\{\begin{array}{c}
(0(l+m), 0(l+m)+i j): \\
i j \in\{0(l+m), 0(l+3 m), 2(l+m), 2(l+3 m)\}
\end{array}\right\} \\
\left\{\begin{array}{c}
\{(n l, n l+i j): i j \in\{1 l, 1(l+2 m), 3 l, 3(l+2 m)\}\} \cup \\
(n)(l+m), n(l+m)+i j):
\end{array}\right. \\
i j \in\{1(l+m), 1(l+3 m), 3(l+m), 3(l+3 m)\}
\end{array}\right\}
\end{array}\right\} .
\end{aligned}
$$

Theorem 12. For all positive integers $m, n \geq 2$, there exists an $O D C$ of $K_{2 m n, 2 m n}$ by $K_{2, m} \cup K_{2, m(n-1)} \cup(3 m n-4) K_{1}$.

Proof. Since $v\left(K_{1, m}\right)$ and $v\left(K_{1,2} \cup K_{2, n-1}\right)$ are symmetric starter vectors, then $v\left(K_{1, m}\right) \times v\left(K_{1,2} \cup K_{2, n-1}\right)$ is a symmetric starter vector with respect to $\mathbb{Z}_{m} \times \mathbb{Z}_{2 n}$ (Theorem 4 ), and the resulting symmetric starter graph has the following edges set:

$$
\begin{aligned}
& E\left(K_{2, m} \cup K_{2, m(n-1)}\right) \\
&=\bigcup_{\alpha=0}^{m-1}\{(01, \alpha \beta),(00, \alpha 0),(0 n, \alpha 0), \\
&\quad(0(n+1), \alpha \beta): 2 \leq \beta \leq n\} .
\end{aligned}
$$

Lemma 13. For any positive integer $m$, there exists an ODC of $K_{6 m, 6 m}$ by $K_{2,3 m} \cup(9 m-2) K_{1}$.

Proof. Since $v\left(K_{1, m}\right)$ and $v\left(K_{2,3}\right)$ are symmetric starter vectors, then $v\left(K_{1, m}\right) \times v\left(K_{2,3}\right)$ is a symmetric starter vector with respect to $\mathbb{Z}_{m} \times \mathbb{Z}_{6}$ (Theorem 4 ), and the resulting symmetric starter graph has the following edges set:

$$
E\left(K_{2,3 m}\right)=\bigcup_{\alpha=0}^{m-1}\{(00, \alpha \beta),(03, \alpha \beta): \beta \in\{0,1,5\}\} .
$$

\section{Conclusion}

In conclusion, the known symmetric starters are used as ingredients for the cartesian product construction to obtain new symmetric starters which are $m K_{2,2 n} \cup 2 m(3 n-$ 1) $K_{1}, m K_{4,4} \cup 24 m K_{1}, 2 m K_{4,4} \cup 48 m K_{1}, n m K_{4,4} \cup 24 m n K_{1}$, $K_{1,2 m} \cup K_{1,2 m(n-1)} \cup 2(m n-1) K_{1}, K_{1,4} \cup K_{1,4(n-1)} \cup K_{1,4(m-1)} \cup$ $K_{1,4(m-1)(n-1)} \cup 4(m n-1) K_{1}, m K_{2,4} \cup m K_{2,4(n-1)} \cup 4 m(3 n-$ $1) K_{1}, K_{2, m} \cup K_{2, m(n-1)} \cup(3 m n-4) K_{1}$, and $K_{2,3 m} \cup(9 m-2) K_{1}$.

\section{References}

[1] H.-D. O. F. Gronau, M. Grüttmüller, S. Hartmann, U. Leck, and V. Leck, "On orthogonal double covers of graphs," Designs, Codes and Cryptography, vol. 27, no. 1-2, pp. 49-91, 2002.

[2] S. Hartmann and U. Schumacher, "Orthogonal double covers of general graphs," Discrete Applied Mathematics, vol. 138, no. 1-2, pp. 107-116, 2004.
[3] R. El-Shanawany, Hans-D.O. F. Gronau, and M. Grüttmüller, "Orthogonal double covers of $K_{n, n}$ by small graphs," Discrete Applied Mathematics, vol. 138, no. 1-2, pp. 47-63, 2004.

[4] M. Higazy, A study of the suborthogonal double covers of complete bipartite graphs [Ph.D. thesis], Menoufiya University, 2009.

[5] R. Scapellato, R. El-Shanawany, and M. Higazy, "Orthogonal double covers of Cayley graphs," Discrete Applied Mathematics, vol. 157, no. 14, pp. 3111-3118, 2009.

[6] R. Balakrishnan and K. Ranganathan, A Textbook of Graph Theory, Universitext, chapter 1, Springer, New York, NY, USA, 2nd edition, 2012.

[7] R. El-Shanawany, Orthogonal double covers of complete bipartite graphs [Ph.D. thesis], Universität Rostock, 2002. 


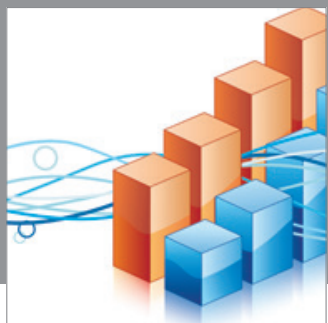

Advances in

Operations Research

mansans

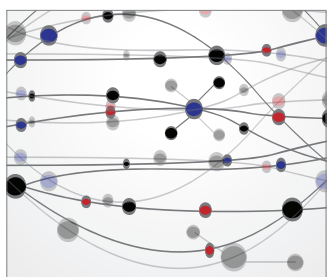

The Scientific World Journal
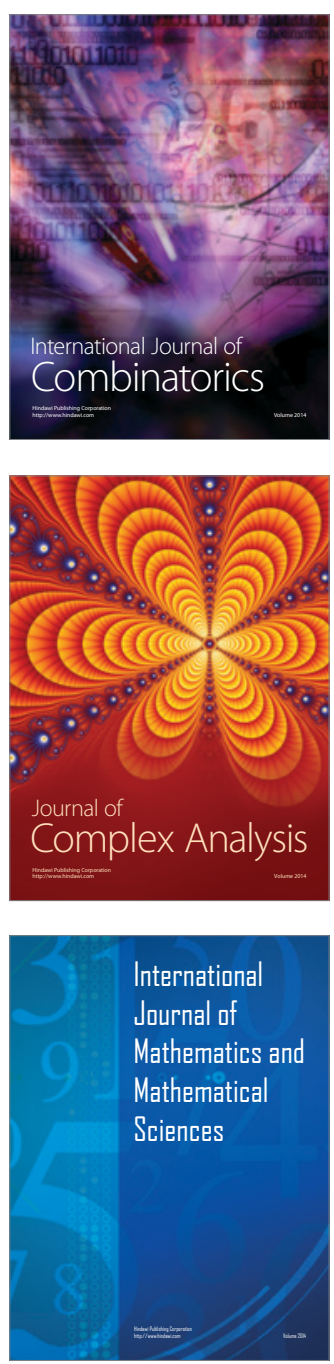
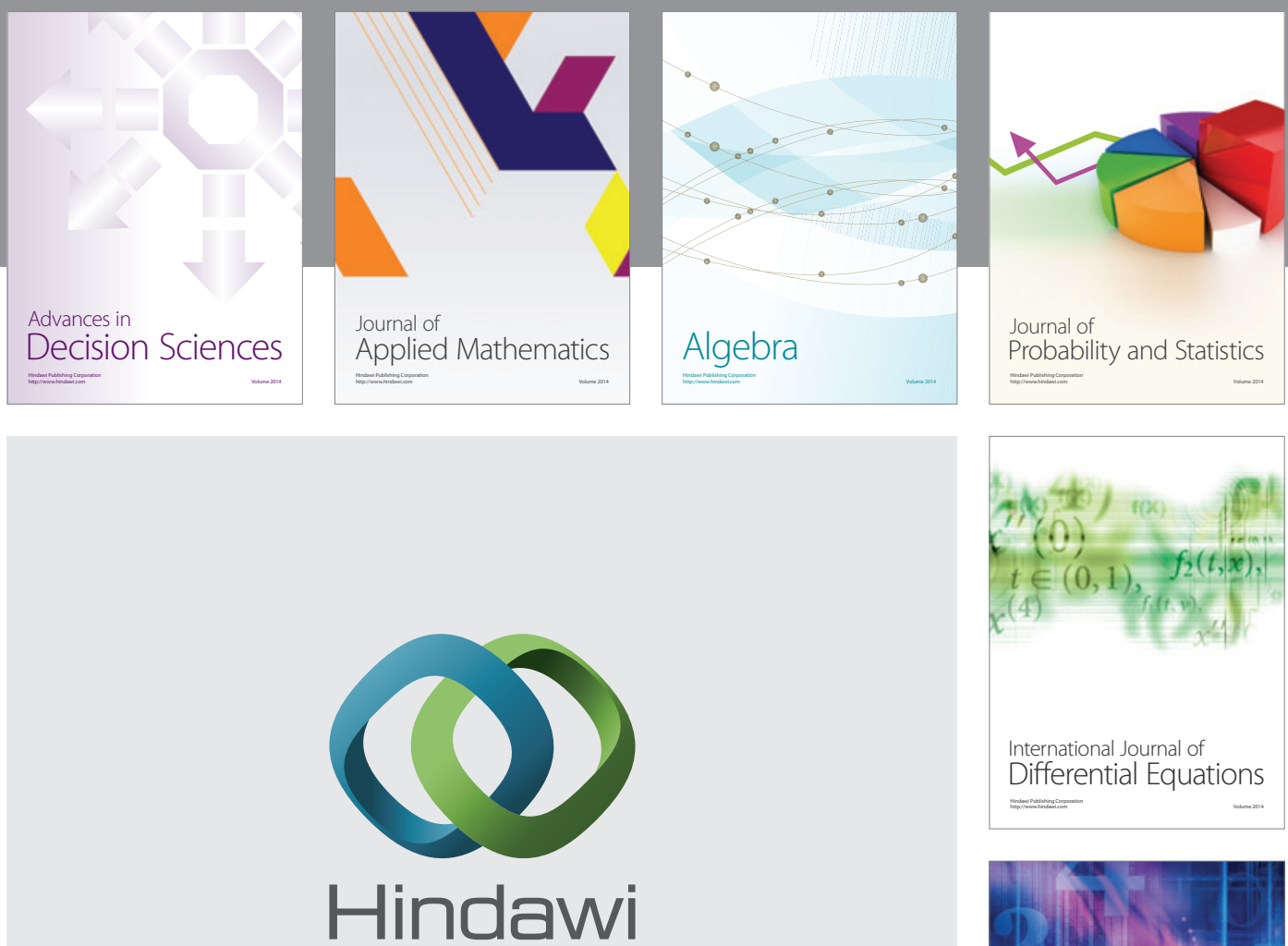

Submit your manuscripts at http://www.hindawi.com
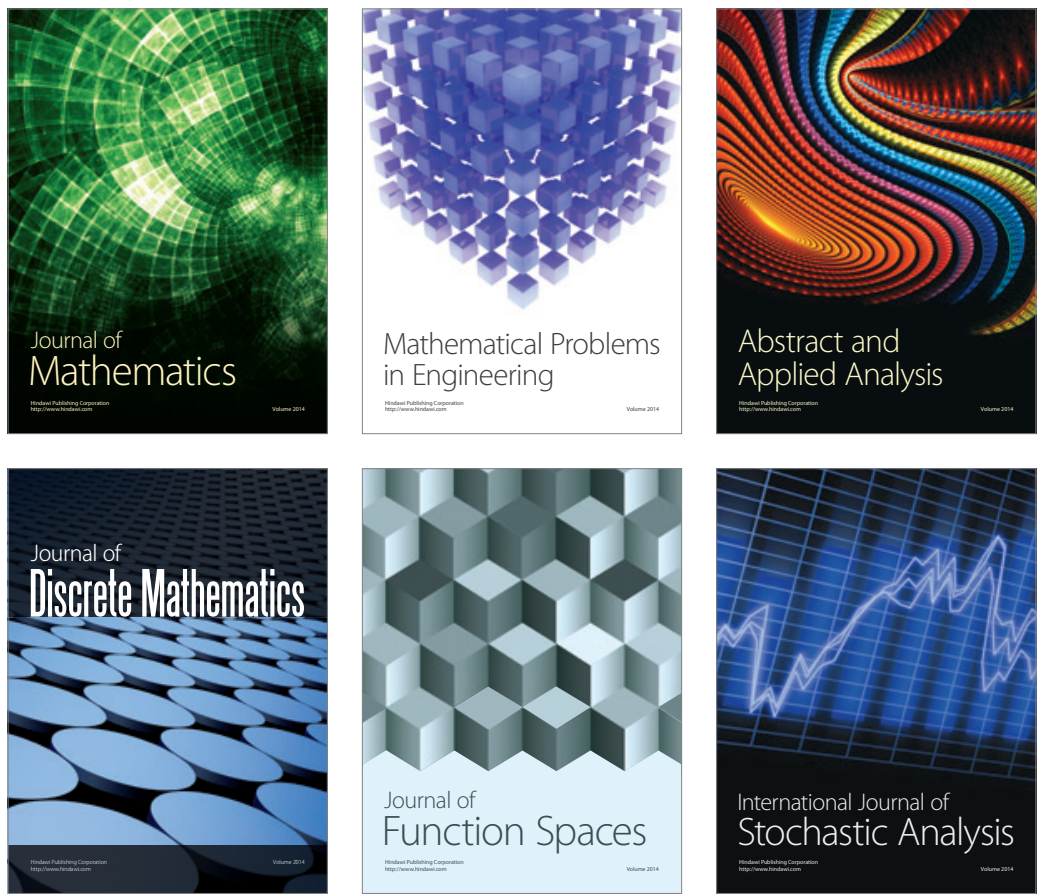

Journal of

Function Spaces

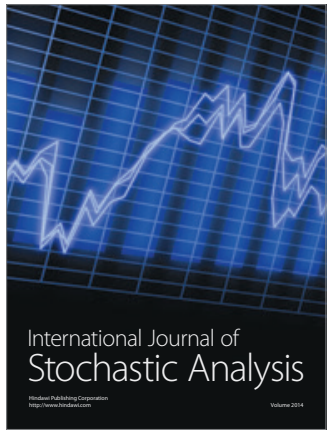

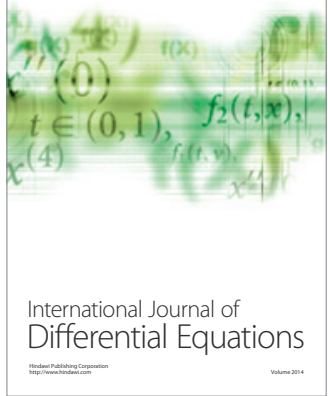
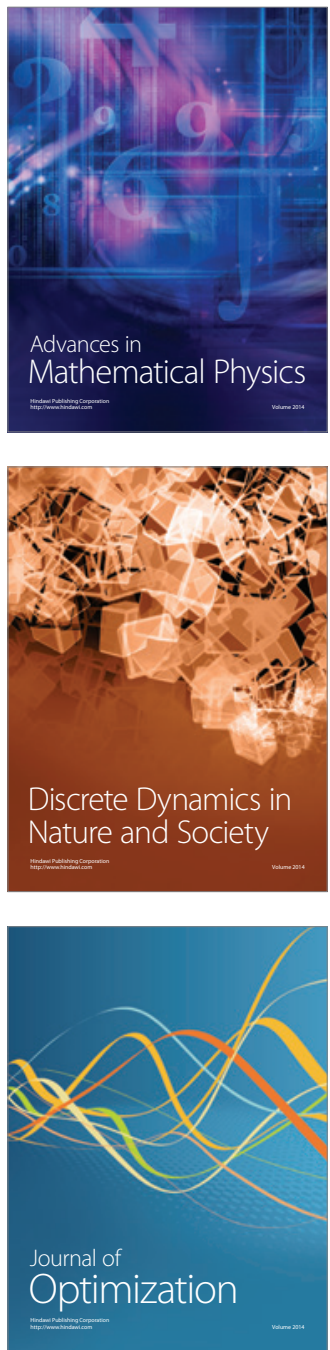